Aus dem I. Anatomischen Institut in Wien (Prof. Tandere).

\title{
Anatomische Untersuchungen über zwei Fälle von Perückenbildung beim Reh.
}

\author{
Von \\ Dr. Marianne Stein, \\ Assistent des Instituts. \\ Mit 4 Figuren im Text. \\ Eingegangen am 25. Januar 1914.
}

Unter den Säugern zeichnen sich die Cerviden durch gute Entwicklung der männlichen und weiblichen sekundären Geschlechtscharaktere aus. Aus diesem Grunde haben auch TandLer und Grosz bei ihren Studien über die sekundären Geschlechtsmerkmale Cerviden für ihre Experimente gewäblt. Die Auffälligkeit dieser Geschlechtscharaktere hatte auch zur Folge, daß Störungen an ihnen schon seit langer Zeit beobachtet wurden. Doch handelt es sich hierbei meist um Zufallsbefunde von Jägern und fast nie nm zielbewußte morphologische Untersuchangen. RoErig, der einige sehr bekannte Arbeiten iiber Geweihanomalien der Cerviden publizierte, zählt eine Reihe von Faktoren auf, die Geweihabnormitäten hervorrufen und führt auch für jede Art der Deformität Beispiele an. Ziemlich ausführlich behandelt er die Kapitel der Reduktion oder Deformität des Geweihes infolge von Verletzungen der Extremitäten oder des Schädels und von Erkrankungen der inneren Organe oder des Gesamtkörpers; hauptsächlich beschäftigt er sich jedoch mit den Korrelationen zwischen Fortpflanzungsorganen und Geweihbildung. Obwohl er zur Klarlegung dieser Beziehungen eine große Menge von Beispielen bringt, kann er schon deshalb nicht zu verläßlichen Resultaten kommen, weil er einen großen Teil der Fälle, die er als Beispiele heranzieht, nicht selbst gesehen hat, sondern sich auf die meist recht oberflächlichen Angaben von andern, größtenteils Jägern, berufen muß, deren Beschreibungen anatomische Grundlagen entbehren. 
Es wird daher begreiflich erscheinen, wenn ich im nachfolgenden zwei mir von Herrn Professor Tandler tiberlassene Fälle von Pertickenbildung beim Reh beschreibe, deren genane Untersuchung möglich war. Den ersten Fall verdanke ich Herrn Professor v. Lonenz, Direktor des k. k. Hofmuseums in Wien, der die Freundlichkeit hatte, das Becken eines Rehes mit dem Genitale dem Institute zur Verfugung zu stellen und die Perïcke dieses Tieres leihweise zu überlassen; den zweiten Fall danke ich der Liebenswirdigkeit des Herrn Dr. Heinde, der uns das Genitale und Photographien der Perticke eines Rehes übermittelte.

Beim ersten Fall sieht man am Schädel des Tieres ein mächtiges,

Fig. 1.

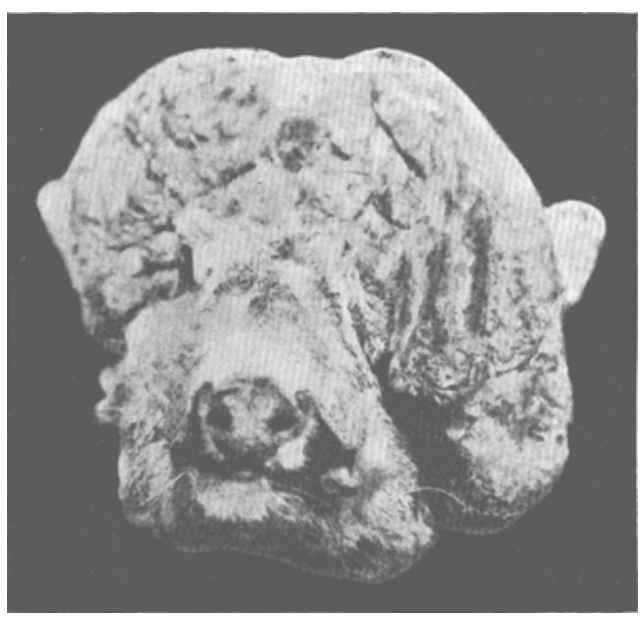

diesem mützenartig aufsitzendes Gebilde; es ist weich, ubberall mit kurzen Haaren bedeckt, und zerfällt durch seine oberflächliche Zerklüftung in einzelne Lappen. Dureh seinen Sitz, seine Konsistenz und seine Behaarung ist dieses Gebilde als ein Periuckengeweih charakterisiert (Fig. 1). Es sitzt, wie Perticken im allgemeinen, der Stirnregion auf und hat daher eine im Verhältnis zu seiner Größe schmale Basis.

Von den sich seitlich ausbreitenden Lappen reicht der rechte bis an das Auge, während der linke das Auge dieser Seite vollkommen bedeckt und sich über den Unterkieferwinkel nach hinten und unten erstreckt. Ferner zeigt sich, daß von der Schädeldecke an der entsprechenden Stelle zwei Stirnzapfen entspringen, deren Länge nicht feststellbar ist, weil das Präparat geschont werden muß.

Perïckenbildung bei Rehböcken ist keine sehr seltene Geweihanomalie, auffallend ist jedoch das Vorhandensein einer Perücke in diesem Falle, da es sich um ein weibliches Tier handelt. Geweihbildung bei Rehgeißen ist einwandfrei bisher selten beobachtet worden. RoERIG hat bei geweihtragenden Cervidenweibchen auf das häufige Vorhandensein von Atrophie oder Erkrankung der Genitalien oder 
Hermaphroditismus hingewiesen, macht aber gerade von jenen Fällen, in welchen es zur Perückenbildung gekommen ist, nur fluchtige Angaben ïber das Verhalten des Genitales, spricht iberhaupt nur zweimal von einem Perïckengeweih, einigemal von einem perïckenartigen Geweih. Diese seltene Erwähnung der Perückenbildung bei weiblichen Cerviden läßt bei dem großen Material Roerigs er hat ungefähr 70 Fälle beschrieben - auf sehr seltenes Vorkommen dieser Anomalie bei Weibchen schließen. Um so erwünsehter war es mir, daß sich mir Gelegenbeit bot, das Genitale dieser Rehgeiß makro- und mikroskopisch zu untersuchen.

Die Beckenorgane in situ betrachtet, zeigen in Bezug auf Form und Ausdehnung des Carum Douglasi keinen Unterschied gegen die eines normalen Weibchens. Der Uterus reicht etwa handbreit uber den kranialen Symphysenrand in die Abdominalhöhle hinein. Die beiden Hörner sind plump und im Verhältnis zum Corpus uteri kurz. Sie sind nicht eingedreht, sondern verlaufen, besonders das linke, fast vollkommen transversal. Corpus und Cornua sind platt und von derber Konsistenz. Die Tuben sind im aterinen Anteil schwach geschlängelt, der laterale Abschnitt zeigt eine deutlich ausgeprägte, brïckenförmig ausgespannte Mesosalpinx, so daß eine dorsalwärts offene Ovarialtasche zustande kommt. Die Ovarien haben eine glatte Oberfläche und gelbgraue Farbe. Ihr Längsdurchmesser ist etwa $1 \mathrm{~cm}$, ibr Breitendurchmesser $7 \mathrm{~mm}$ und ihr Dickendurchmesser 3-4 mm. Besonders vorspringende Auftreibungen, wie sie einem reifenden Follikel oder einem Corpus luteum entsprechen, fehlen an beiden Ovarien. Die Ligamenta propria ovarii sind als weiße, derbe Falten sichtbar. Das Ligamentum teres uteri liegt jederseits in einer flïgelförmigen Duplikatur des Peritoneums, hat einen geschlängelten Verlauf und läßt sich deutlich bis zum Inguinalkanal verfolgen. Auffallend stark sind die Gefäße entwickelt. Die Vasa ovarica werfen eine mächtige plumpe Gefäßfalte auf, die Vasa uterina sind im Ligamentum latum als kräftiger Gefäßstrang wabrnehmbar. Das äußere Genitale verhält sich wie beim normalen Tiere.

Entnimmt man das Genitale dem Becken und eröffnet die Vagina, so gelangt man aus einem weiten Vestibulum vaginae in einen engen, äußerst starkwandigen mit der Urethra fest verbundenen Anteil der Vagina; kranial von diesem Abschnitt erweitert sich die Vagina plötzlich und wird erst am Fornix wieder schmäler. Von den seitlichen Ausladungen ziehen zarte Falten gegen die Portio uteri. Diese zeigt ein zerklüftetes Aussehen, besteht aus größeren und kleineren. 
Wülsten, zwischen welchen sich der Eingang in den Uterus befindet. Der Uterus ist bis za seiner Teilungsstelle in die beiden Cornua etwa $10 \mathrm{~cm}$ lang. Eröffnet man ihn, so sieht man, daß er in seiner oberen Hälfte durch ein Septum in zwei Anteile geschieden ist. Die Schleimbaut ist glatt, in der rechten Hälfte gelblich, in der linken dunkelrotbraun gefärbt. Die Innenfläche weist eine Reihe gegen die Nachbarschaft deutlich abgegrenzter Prominenzen auf, welche, wie noch gezeigt wird, Karunkel darstellen. Sie sind an der Wand des Carum uteri folgendermaßen verteilt: am peripheren Ende eines jeden Hornes sitzt eine Karunkel der hinteren Wand auf, die nächste erhebt sich jederseits an der lateralen Wand des Cornu, das dritte Paar sitzt auf der Vorderwand des Corpus, ebenso das vierte Paar, dessen rechte Karunkel allerdings etwas mehr gegen die laterale Wand zu inseriert.

Diese Erhebungen sind mit ihrer Längsachse parallel der Längsachse des Uterus eingestellt und sitzen zumeist mit breiter Basis der Unterlage auf. Nur eine des linken Hornes ist gestielt. Die Länge der Karunkeln beträgt durchschnittlich $2 \mathrm{~cm}$, ihre Breite $3 \mathrm{~mm}$ bis $1 \mathrm{~cm}$, die Dicke $2-7 \mathrm{~mm}$. Sie haben in der rechten Uterushälfte eine gelbe Farbe und glatte Oberfläche, auf der linken Seite sind sie, ähnlich wie die übrige Schleimhaut dieser Seite, dunkler gefärbt; die rötlichbraune Farbe ist jedoch keine gleichmäßige, sondern von helleren Flecken durchbrochen. Die Oberfläche zeigt einen feinen, körnigen Belag. Die eben beschriebenen Prominenzen sind nach ihrer Lage, Form und Farbe, sowie nach dem Aussehen ibrer Oberfläche als Karunkel anzusehen und wurden daher unter diesem Namen in die Beschreibung eingeführt. Über das Verhalten der Karunkeln beim Reh besitzen wir wohl Angaben von STRAHL, doch beziehen sich diese nur auf den graviden Uterus. Eine Beschreibung der Karunkeln im Rehuterus vor oder nach der Gravidität konnte ich in der mir zur Verfügung stehenden Literatur nicht auffinden. Der makroskopische Befund am Uterus und an den uterinen Gefäßen zeigt, daß es sich in diesem Falle um einen puerperalen Uterus handelt, doch sind mir keine genaueren Daten tiber die puerperalen Veränderungen der Karunkeln beim Reh bekannt, so daß es mir nicht möglich ist, auf Grund des erhobenen Befundes, eine Schätzung vorzunehmen, welche Zeit vom Partus bis zur Erlegung des Tieres vergangen war. Unter Benuitzung der Angaben von HrLty, welcher die Veränderungen der Karunkeln der Kuh während und nach der Gravidität genau beschreibt, und von STRAHL und MaRtrs, die beim Schafe die puerperale Involution des Uterus 
untersuchten, kann man wohl per analogiam schließen, daß die hier beschriebenen Karunkeln einige Wochen post partum sind. Der Fötus scheint im linken Uterushorne gesessen zu haben, da die Karunkeln dieser Seite weniger rückgebildet sind als auf der rechten, teilweise sogar noch gestielt und mit einem Belage bedeckt sind. Auch die geringere Krümmung des Cornu sinister spricht für linksseitige Gravidität.

Läßt also schon der makroskopische Befund - Größe des Uterus und der uterinen Gefäße, Verhalten der Karunkeln, Form der Portio - auf eine abgelaufene Gravidität schließen, so wird diese Annahme durch die mikroskopische Untersuchung bestätigt. Ein Querschnitt durch ein Uterushorn zeigt großen Gefäßreichtum der Uteruswand, besonders an der dem Ansatz des Parametrium zugekehrten Seite des Uterushornes. Am antiparametralen Rande sind die Gefäße kleiner. Die Muskelschicht ist gut entwickelt, stellenweise reichlich von Gefäßen durchsetzt und vom Endometrium deutlich abgegrenzt, letzteres ist breit und läßt ein äußeres drüsenreiches, ein mittleres vorwiegend bindegewebiges and ein inneres gefäßreiches Stratum erkennen. Eine von den makroskopisch beschriebenen Karunkeln ist am Querschnitt getroffen. Sie besteht aus einer locker gewebten, zell- und gefäßreichen Masse, von den Schichten der tibrigen Schleimhaut fehlt die Drüsenschicht. Das Epithel ist infolge der ungenügenden Konservierung nur an wenigen Stellen erbalten. Die erwähnte Karunkel erweist sich aus folgenden Gründen als puerperale: Sie ist sehr gefäßreich, besteht aus lockerem Gewebe, dessen Zellen auch nicht in der Randzone dichter stehen, und sitzt der Unterlage mit einer stielartigen Basis auf. Da aber die Oberfläche der Karunkel konvex und ziemlich glatt ist, die Gefäße zumindest in jenem Anteil der Karunkel, in welchem sie der Uterusinnenfläche aufsitzt, dickwandig und in Obliteration befindlich sind, ist der Partus nieht unmittelbar voransgegangen. An Schnitten durch eine Karunkel des rechten Uterushornes sieht man, daß diese der Wand mit breiter Basis aufsitzt; ferner sind in ihr weniger und kleinere Gefäße vorhanden, als in der oben beschriebenen der linken Seite, so daß auch mikroskopisch die Karunkeln der beiden Uterushälften ein verschiedenes Aussehen zeigen.

Zur mikroskopischen Untersuchung der Ovarien wurde das linke fast vollkommen geschnitten und die Schnitte mit Haemalaun Eosin und nach verschiedenen Methoden der Bindegewebsfärbung gefärbt. Man sieht zunächst eine Reibe von großen, dickwandigen Gefäßen, die sich in der ganzen Ovarialsubstanz ausbreiten. Die 
Marksubstanz ist reichlich und zeigt ein streifiges Gefüge. Corpora albicantia in Form von wellenförmig verlaufendem hyalinen Bindegewebe sind besonders deutlich am VAN GIEson-Präparate sichtbar. Die Rindensubstanz enthält nur sehr wenig Primordialfollikel, anch GraAfsche Follikel sind spärlich. Der größte Teil des Ovariums wird von zwei Gebilden eingenommen: einem großen, fast sprungreifen, allerdings tiber die Oberfläche nicht vorragenden mit Cumulus oophorus versehenem Follikel und einem länglichen, durch Bindegewebe gut abgegrenzten Körper. An seinem peripheren Pol verliert er die Begrenzung und öffnet sich gegen die Oberfläche. - Er hat ein zartes Bindegewebegerist, in dessen Maschen einzelne Zellen und Zellinseln liegen. Das Protoplasma dieser Zellen ist dunkel und granuliert, der Kern groß und exzentrisch gelegen. Sie liegen entweder einzeln oder in kleinen Gruppen beisammen. Diese Zellen, die aller Wahrscheinlichkeit nach Luteinzellen sind, zeigen, daß es sich hier um ein Corpus luteum im Beginn seiner Rückbildung handelt. Außerdem sieht man bei schwacher Vergrößerung eine Reihe von Quer- und Längsschnitten durch strangartige Gebilde, welche sich von ihrer Nachbarschaft durch ihre lebhafte Färbung abheben. Bei stärkerer Vergrößerung zeigt sich, daß diese Stränge stellenweise ein äußerst feines, mit Endothel ausgekleidetes Lumen besitzen, während an andern Durchschnitten eine solche Lichtung nicht nachweisbar ist. Es handelt sich, wie die genauere Untersuchung zeigt, um obliterierende Gefäße mit besonders dicken Wandungen und starken Schlängelungen.

Vom rechten Ovarium wurde nur eine Anzahl von Querschnitten verfertigt. Auffallend ist der Reichtum an großen, besonders starkwandigen, mit Intimaverdickungen versehenen Gefäßen. Die Marksubstanz nimmt den größten Teil des Ovariums ein und hat eine streifige Struktur. In der Rinde sind etwas mehr Primärfollikel als im linken Ovarium zu sehen, doch fehlen größere Follikel und Corpora lutea völlig. Hingegen findet man auch hier einige Bildungen, die man nach ihrer Form und Struktur für Corpora albicantia ansehen muß.

Der Vergleich des mazerierten Beckens mit dem eines normalen männlichen Rehes ergibt ein typisches weibliches Becken, welches durch vollkommene Verknöcherung der Symphyse als das eines älteren Individuums charakterisiert ist. Es bestebt ein weiter Arcus pubis. Das Kreuzbein ist kurz und breit, seine Breite verhält sich zur Länge wie 51/2:7. Beim Männchen ist das Verhältnis $4 \frac{1}{2}: 7$. Die Facies auriculares zeigen bei der Perückengeiß einen nahezu parallelen 
Verlauf. Die Darmbeinteller sind flacher und niedriger als beim Männchen. Bei gleicher Länge der Becken - gemessen am Abstand der Spina iliaca anterior superior vom oberen Rand der Symphyse ist die Weite des Beckens der Peruckengeiß durchschnittlich um $1 \mathrm{~cm}$ größer. Zum Beispiel beträgt die Entfernung der durch die Ausbuchtung der Fossae acetabuli an der Innenseite des Beckens hervorgerufenen Prominenzen 5,3 bzw. 4,4 cm. Der Abstand der beiden Spinae ischiadicae beträgt $5,4 \mathrm{bzw} .4 \mathrm{~cm}$. Die Conjugata vera ist um $1 \mathrm{~cm}$ länger als beim Männchen. Somit besitzt das Becken der Perückengeiß sümtliche sekundäre Geschlechtscharaktere des weiblichen Beckens, ein Verhalten, das nach den Befunden an Uterus und Ovarien, funktionierenden Organen, nicht, zu verwundern ist.

Hiermit wird die Angabe Roenigs, daß Geweihe bei fruchtbaren weiblichen Cerviden atypische sind, bestätigt. Ansätze von Geweihen sind bei weiblichen Rehen häufig beschrieben worden. TandLen und Grosz erklären dieses Vorkommen dadurch, daß die Geweihe als Speziesmerkmale beiden Geschlechtern zugekommen sind und erst sekundär als Geschlechtsmerkmale modifiziert wurden. Daß beide Geschlechter ursprünglich Geweihe getragen haben, behauptet auch Alston, indem er von der Ansicht ausgeht, daß die Geweihe als Waffen gegen gemeinsame Feinde beiden Geschlechtern ursprünglich zugekommen seien. Die Ansichten von Tandler und Grosz über die Phylogenese des Geweihes wird noch gestützt durch einen entwicklungsgeschichtlichen Befund Tanduens. Dieser konnte nachweisen, daß sich regelmäßig auch beim weiblichen Hirschfötus eine der männlichen analoge Geweihanlage vorfindet. Nach den eben erwähnten Befunden wäre es wohl zu verstehen, daß Rebgeiße manchmal Geweihe tragen, unerklärt bleibt aber das Auftreten eines Perückengeweihes beim weiblichen Tier. Beim Rehbock entwickelt sich ein Perückengeweih im AnschluB an die Kastration. Über die Art und Weise dieser Entwicklung kann hier nichts Näheres ausgesagt werden, es muß vielmehr auf die Arbeiten von TANDLER und Grosz, welche die einschlägige Literatur berücksichtigen, hingewiesen werden. Die experimentellen Untersuchungen dieser Antoren haben gelehrt, daß die Geweihbildung und ihr normaler Ablauf von der innersekretorischen Wirksamkeit der Hodenzwischenzellen (LEXDIGsche Zellen) abhängig sei. $\mathrm{Da}$ es während der Gravidität, wie vielfach behauptet wird, auch za einer Unterfunktion des Ovars im allgemeinen, also auch zu einer solchen der innersekretorischen Elemente kommt, so liegt die Idee nahe, daß die Perückenbildung in diesem 
Falle auf die Unterfunktion des Orariums zurückzufuhren sei, die Rehgeiß schon früher ein Geweih getragen babe, welches zur Zeit der Gravidität zu einer Perüeke umgestaltet wurde.

Der zweite Fall betrifft ein Reh, welches uns von Dr. Heindu überlassen wurde. Das Genitale war aus dem Becken herausgeschnitten.

Die Perücke selbst konnte ich nicht sehen; auf den in liebenswürdiger Weise von Dr. HEIndL angefertigten Photographien sieht man am Kopfe des Tieres ein Perückengeweib, welches sich von dem früher beschriebenen vor allem in seiner Form unterscheidet (Fig. 2). Dem Bilde und ergänzenden Mitteilungen von Dr. Heinde ist zu

Fig. 2.

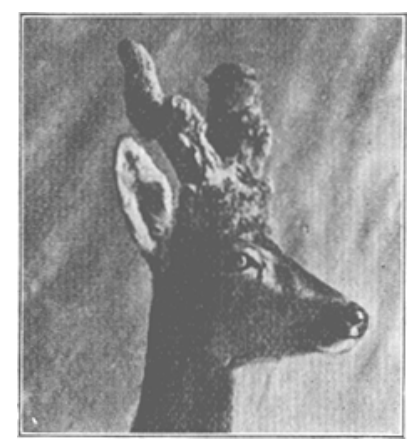
entnehmen, daß die rechte Stange die Form eines Sechsergeweihes hat, die linke plumper und ohne Sprossen ist. Das Geweih soll nur wenig verknöcherte Substanz enthalten haben, im frischen Zustande weich und an einigen Stellen behaart gewesen sein. Mit diesem Geweih wurde das Reh schon seit 5 Jahren beobachtet. Da die Stimme des Tieres eine für das Weibchen charakteristische Höhe hatte, der ganze Habitus ein weiblicher und der Hoden von außen nicht tastbar war, so war es nabeliegend, das Tier fur ein Weibchen zu halten. Die oberflächliche Betrachtung des inneren Genitales könnte die Vermutung bestätigen (Fig. 3). Hinter der Blase sieht man ein queres, aus einer Peritonealduplikatur gebildetes Septum, dessen freier Rand von einem strangförmigen Gebilde eingenommen wird. Das Septum endet lateral mit einem unregelmäßigen Schnittrand und sieht wie ein Ligamentum latum aus. Die beiden Stränge konvergieren medialwärts, bis sie so nahe aneinander liegen, daß sie wie ein einheitlicher Strang erscheinen und verlaufen so in der Medianebene beckenwärts. An der hinteren Fläche der transversal verlaufenden Falte liegt rechterseits ein etwa $5 \mathrm{~mm}$ langes, $2 \mathrm{~mm}$ breites Körperchen, zu dem von oben her einzelne Gefäße ziehen. Nach Aussehen und Gefäßrerteilung könnte dieses Gebilde ein Ovarium darstellen. Auf der linken Seite ist ein solcher Körper nicht vorhanden 1).

1) Als Nebenbefund wäre noch eine walnußgroße, der Blasenhinterfläche anliegende, prall gefüllte Cyste, die zwischen beiden Peritonealblittern gelegen ist, zu erwähnen. 
Erst die genauere Präparation zeigt, daß es sich hier nicht um ein weibliches Individuum handelt. Die beiden oben erwähnten dünnen Stränge lassen sich hinter der Blase kaudalwärts verfolgen, verschwinden dann zwischen dem Blasenhals und den hinter demselben gelegenen derben, länglichen, gut begrenzten Körperchen der Prostata, und stellen die Ductus deferentes dar, welche allem Anschein nach in einem atrophischen Zustand sind, da sie viel düner

Fig. 3.

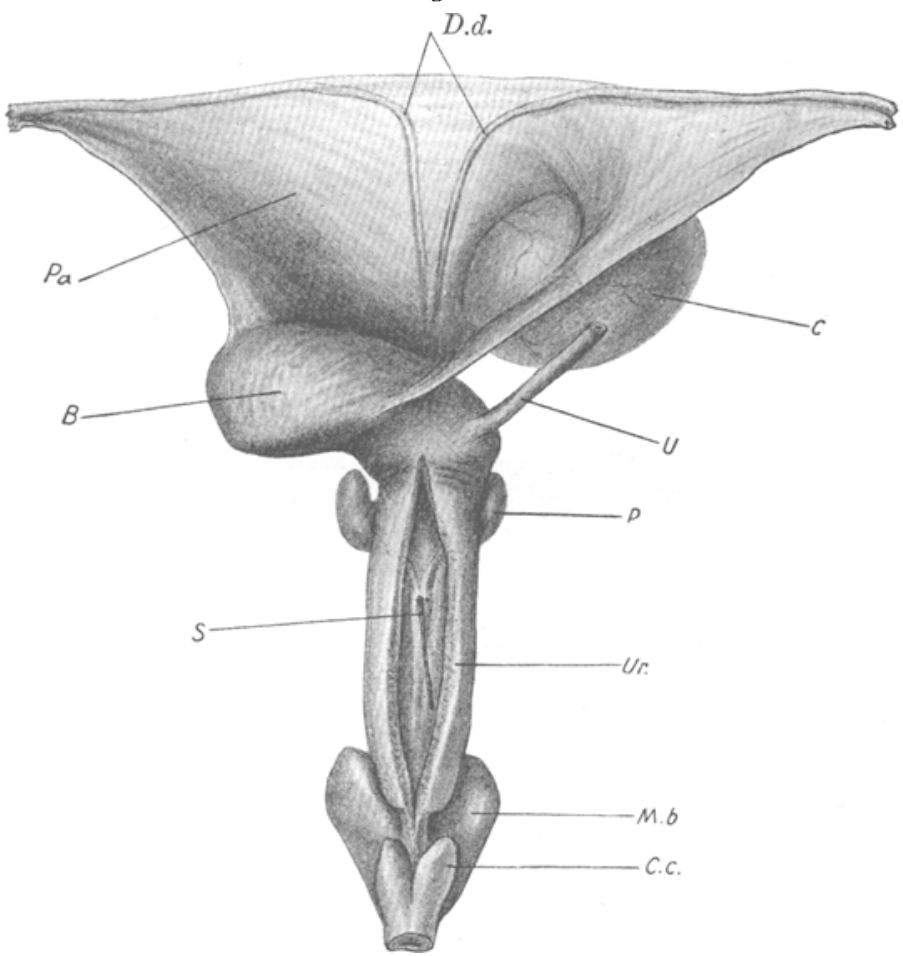

B. Blase, C. Cyste, C.c. Corpus cavernosum, D.d. Ductus deferens, M.b. Musculas bulbocavernosus, $P$. Prostata, $P d$. Peritonealduplikatur, $S$. Sonde im Ductus deferens, $U$. Ureter, $U_{r}$. Urethra.

sind als die normalen. Verfolgt man diese Stränge hodenwärts, so findet man ihr Ende an dem Schnittrande der transversal gestellten Bauchfellduplikatur.

Die Blase setzt sich in eine lange und dickwandige Pars prostatica urethrae fort, auf welche eine regelmäßig gebaute Pars membranacea folgt, an deren distalem Ende weiche, in der Medianebene einander berïhrende Körper gelegen sind - besonders mächtig entwickelte Masculi bulbo-cavernosi. Glandulae Cowperi, die an dieser Stelle 
liegen sollten, waren nicht za sehen. Die sich anschließende Pars cavernosa urethrae ist von zwei, mit je einer länglichen derben Anschwellung beginnenden Corpora cavernosi begleitet, zeigt also normales Verbalten. Der Penis ist etwa $20 \mathrm{~cm}$ lang und scheint normal entwickelt zu sein. Das Praeputium endet auf der Höhe einer vor den Zitzen gelegenen stumpfen Prominenz.

Öffnet man Blase und

Fig. 4.

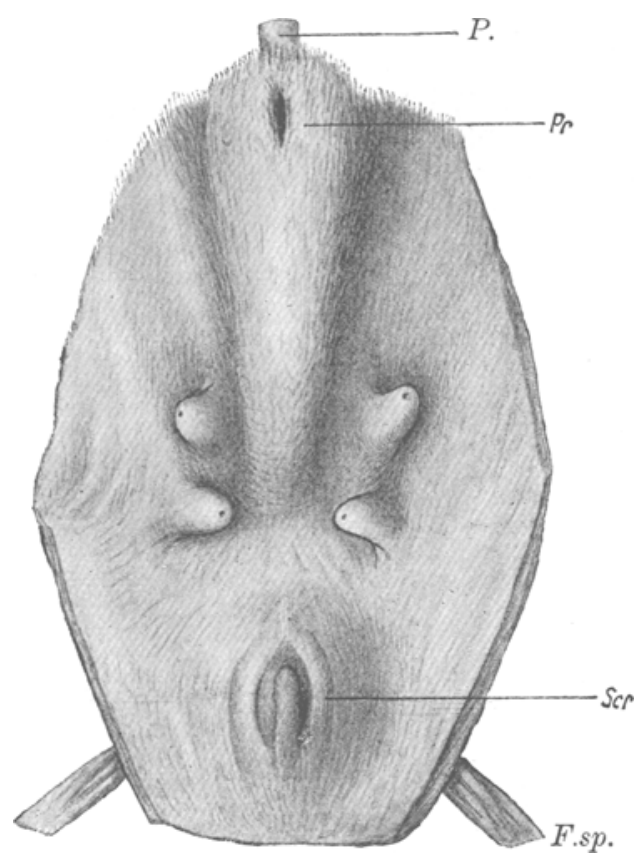

F.sp. Funicalus spermaticus, $P$. Penis, $P r$. Praeputium, Scr. Serotum. Urethra, so erscheinen die an normaler Stelle gelegenen Uretermündungen und an der dorsalen Wand der Urethra, beiläufig an jener Stelle, an welcher außen die Prostata gelegen ist, eine kleine Prominenz, die zwei schlitzförmige Mündungen trägt. Diese führen in die beiden früher beschriebenen strangartigen Gebilde des Lig. latum. Es handelt sich demnach hier um die Mündungen der Ductus deferentes.

Hinter der Zitzenregion, welche vier längliche, gut ausgebildete Mamillae trägt, erhebt sich in der Medianlinie eine etwa $1 / 2 \mathrm{~cm}$ bohe Prominenz von geringer Breite, die nach einem Verlauf von ungefähr $1 \mathrm{~cm}$ allmählich verstreicht (Fig. 4). Lateral von ihr, durch seichte Furchen getrennt, befindet sich jederseits eine stumpfe Erhebung, deren Haut dünn und gefaltet ist; diese Vorwölbung entspräche dem Serotum. Doch kann man durch ihre Haut keinen Körper, der Form und Konsistenz des Hodens hätte, tasten. Präpariert man jedoch an der Innenseite, so sieht man gegen diese Gegend jederseits einen proximalwärts leider abgeschnittenen Strang verlaufen. An ihm lassen sich eine peritoneale Aussackung, einige gefäßartige Gebilde und Züge quergestreifter Muskulatur nachweisen. Er zieht bis nahe an die oben erwähnte Prominenz der Außenseite 
heran und endet mit einer etwa bohnengroßen Verdickung von derber Konsistenz. Dieser Strang stellt den Funiculus spermaticus, das verdickte Ende wahrscheinlich das Hodenrudiment dar. Es liegt, wie man schon von außen tasten konnte, nicht in der scrotumähnlichen Tasche, sondern höher oben.

Die mikroskopische Untersuchung des Hodenrudiments zeigt weite Hodenkanälchen mit stark verdickter, bindegewebiger Wand. Das Epithel ist in einzelnen Kanälchen einschichtig und hoch, obne daß man Spermatoblasten wahrnehmen könnte. An andern Stellen ist es mehrreihig, nirgends sind Stadien der typischen Spermatogenese wahrnehmbar. Einzelne Kanäle sind mit gleichartigen Zellen erfültt, die wahrscheinlich infolge schlechter Konservierung losgelöste SERTOLIsche Zellen darstellen. Vergleicht man den Hoden dieses Tieres mit dem eines normalen Rehes, so fällt neben Unterentwicklung der Samenkanäle auch das veränderte Aussehen des Zwischengewebes auf. Es ist besonders reichlich entwickelt, besteht jedoch fast nur aus Bindegewebe; Gefäße fehlen nahezu vollständig und auch $\mathrm{Zwischen-}$ zellen sind nicht nachweisbar.

Die Prostata enthält reichlich Muskulatur und Bindegewebe, Drüsen sind jedoch nur in geringer Anzahl vorhanden, die Drüsenelemente der Prostata sind also unterentwickelt.

An Schnitten durch den Körper, der seiner Lage und seinem makroskopischen Aussehen nach ein Ovarium darstellen könnte, läßt sich weder eine Rinden- noch eine Marksubstanz erkennen. Auf eine aus festgefugtem Bindegewebe bestehende Randzone folgt netzförmig angeordnetes Gewebe mit großen, polygonalen Maschen. Dieses Organ besteht demnach hauptsächlich aus Fettgewebe. Orarialsubstanz ist nicht vorhanden.

Ein Querschnitt durch den Funiculus spermaticus zeigt, daß er größtenteils von Fett erfullt ist. Außerhalb des Fettes befinden sich einige Bündel quergestreifter Muskulatur - Musculus cremaster. In der Substanz des Fettes sieht man an einer zirkumskripten Stelle die von mächtigen Bindegewebszägen umgebenen Venen des Plexus pampiniformis und einige Querschnitte starkwandiger Arterien. Der Ductus deferens hat eine auffallend verdickte Wand, die hauptsächlich ans glatter Muskulatur besteht; nur in den inneren Schichten überwiegt das Bindegewebe. Das Zentrum wird von einem Hohlraum eingenommen, dessen Form jedoch nicht iberall eine gleichmäßige ist. Verfolgt man nämlich Querschnitte, so sieht man, daß nicht eine, sondern 3-5 mit mehrreihigem Epithel ausgekleidete öftnungen 
vorhanden sind, die sich nicht deutlich voneinander abgrenzen lassen. Ebenso wie ihre Zahl, ist auch ihre Form und Größe wechselnd.

Schnitte durch die oben beschriebene Peritonealduplikatur mit dem in ihr eingelagerten Strang lassen die Übereinstimmung im Aufbau dieses Stranges mit dem des Ductus deferens erkennen. Auch hier handelt es sich um ein äußerst dickwandiges Rohr mit mehreren kleinen Lumina. Während an einigen Stellen die Querschnitte der einzelnen Kanälchen ziemlich gut voneinander getrennt sind, konfluieren sie an andern und bilden manchmal Aussackungen eines zentralen Kanales. Auch diese gehen stellenweise verloren, so daß man an einigen Schnitten einen einheitlichen mit hohem, mehrreihigem Epithel versehenen Hohlraum sieht. Als solcher ist er allerdings nur auf einer kurzen Strecke zu verfolgen, dann wird die Grenze des Lumens wieder eine unregelmäßige und neue Ausbuchtungen erscheinen. - Der Ductus deferens zeigt also auch hier kein normales Verhalten. Das Epithel ist, wie beschrieben, von dem des Ductus deferens bei funktionierendem Hoden sehr verschieden und erinnert an embryonale Verbältnisse. Auffällig ist die besondere Wanddicke.

Die Analyse des eben beschriebenen Falles ergibt in Kürze folgendes: Nach dem mikroskopischen Befunde an den Keimdrüsen ist das Tier ein männliches. Nach dem Entwicklungsgrad der Keimdrise ist diese als hypoplastische zu bezeichnen, so daß wir hier einen Fall von Eunuchoidismus beim Rehbock vor uns hätten. Wie die genauere Untersuchung zeigt, ist keine Spur von weiblicher Keimdrüse nachweisbar, so daß von Hermaphroditismus nicht die Rede sein kann. Schwieriger zu beantworten ist allerdings die Frage, wieso es zur Periickenbildung gekommen ist. Diesbezüglich wäre das vollkommene Fehlen der Zwischenzellen verwertbar; da die Zwischenzellen nach den Experimenten von Tandere und Grosz, wie schon erwähnt, für Geweihbildung bestimmend sind, so wäre die Perückenbildung durch Mangel an Zwischensubstanz in diesem Falle erklärlich. Mehr läßt sich über den Zusammenhang zwischen Unterentwicklung der Keimdrïse und der abnormen Geweihbildung in diesem Falle nicht sagen, da es nicht bekannt ist, ob das Tier gleich beim ersten Geweihansatz eine Perücke hatte oder nicht.

Interessant ist, daß zumindest das innere Genitale eine gewisse Ähnlichkeit mit jenem besitzt, welches TANDLER und Keller bei zweieiigen verschieden geschlechtlichen $Z$ willingen des Rindes beschrieben haben, allerdings konnten diese Autoren Veränderungen am Becken und am Schädel solcher Tiere im erwachsenen Zustande 
nachweisen. Diese Veränderungen sind den von TandLer und Grosz beim menschlichen Eunuchoidismus beschriebenen analog. Da auch beim Reh Zwillingsgeburten nicht selten vorkommen, so liegt die Idee nahe, daß der hier beschriebene Fall den einen, und zwar den hypoplastischen Zwilling darstellt. Allerdings ist in der Majorität der Fälle der sogenannte weibliche Zwilling der hypoplastische, doch wurden, wie ich einer mündlichen Mitteilung des Herrn Prof. TandLER verdanke, anch männliche hypoplastische $Z$ willinge von ihm und KELLER beobachtet. Eine weitere Klärung wird diese ganze Frage erst durch den Abschluß der Arbeiten von TANDLER und KELLER erhalten.

Die beiden hier beschriebenen Fälle haben nur einen äußerlichen Zusammenhang, gegeben durch die Perückenbildung, sind aber beide, da sie einer genauen anatomischen Untersuchang zugeführt wurden, geeignet, einen Beitrag zu der komplizierten Lehre von den sekundären Geschlechtscharakteren zu liefern. Gerade der Umstand, daß über die sekundären Geschlechtsmerkmale beim Reh trotz der vielen beobachteten Fälle und der daran geknüpften Hypothesen genauere anatomische Untersuchungen fehlen, läßt es wünschenswert erscheinen, möglichst viel und möglichst gut beobachtetes Tatsachenmaterial zu sammeln.

Meinem verehrten Chef, Herrn Professor Tandler, danke ich zam Schlusse bestens für die Überlassung des Materials und die Förderung, die er meiner Arbeit angedeihen ließ.

\section{Literaturverzeichnis,}

Alston, Proceed. Zoologic. Soc. London. 1879 (zitiert nach Roerig).

Grosser, Eihäute und Placenta. Wien 1909.

Hicty, Schweizer Archiv für Tierheilkunde. 1908.

ROERIG, Beziehungen zwischen den Reproduktionsorganen der Cerviden und der Geweihbildung derselben. Arch. f. Entw.-Mech. Bd. 8.

- Geweihentwicklung und Geweihbildung. Ebenda Bd. 10 u. 11.

Gestaltende Korrelationen zwischen abnormer Körperkonstitution der Cerviden und Geweihbildung derselben. Ebenda Bd. 23.

Strahl, Ergebn. d. Anat. u. Entwicklungsgesch. 190כ. Der Uterus post partum. - Über die Semiplacenta multiplex von Cervus elaphus. Anatom. Hft. Bd. 31. Strahl und Martin, Die puerperale Involution des Uteras beim Schaf. Anatomischer Anzeiger. Bd. 32.

TANDler, Zentralbl. f. Physiologie.

Taxdler und Grosz, Die biologischen Grundlagen der sekundären Gesehlechtscharaktere. Berlin 1913.

Turner, Compar. Anat. of the Placenta. Edinburgh 1876 (zitiert nach RoeriG). 